\title{
Fast time-resolved aerosol collector: proof of concept
}

\author{
X.-Y. Yu ${ }^{1}$, J. P. Cowin ${ }^{1}$, M. J. Iedema ${ }^{1}$, and H. Ali ${ }^{1, *}$ \\ ${ }^{1}$ Pacific Northwest National Laboratory, Richland, WA, USA \\ *now at: Arkansas State University, Jonesboro, AR, USA
}

Received: 1 May 2010 - Published in Atmos. Meas. Tech. Discuss.: 1 June 2010

Revised: 19 August 2010 - Accepted: 6 October 2010 - Published: 12 October 2010

\begin{abstract}
Atmospheric particles can be collected in the field on substrates for subsequent laboratory analysis via chemically sensitive single particle methods such as scanning electron microscopy with energy dispersive $\mathrm{x}$-ray analysis. With moving substrates time resolution of seconds to minutes can be achieved. In this paper, we demonstrate how to increase the time resolution when collecting particles on a substrate to a few milliseconds to provide real-time information. Our fast time-resolved aerosol collector ("Fast-TRAC") microscopically observes the particle collection on a substrate and records an on-line video. Particle arrivals are resolved to within a single frame (4-17 ms in this setup), and the spatial locations are matched to the subsequent single particle analysis. This approach also provides in-situ information on particle size and number concentration. Applications are expected in airborne studies of cloud microstructure, pollution plumes, and surface long-term monitoring.
\end{abstract}

\section{Introduction}

The development of the Fast Time-Resolved Aerosol Collector (Fast-TRAC) was driven by the need to probe dust within microstructures of clouds from a traversing aircraft, though it has applications to many other areas, which are discussed later. Cloud microstructure is often on the scale of $1 \mathrm{~m}$ or less (Stevens et al., 2003). If traversed by an aircraft flying at $150 \mathrm{~m} / \mathrm{s}$, a time resolution of $\sim 7 \mathrm{~ms}$ covers $1 \mathrm{~m}$ of flight. Existing cloud probes such as the cloud imaging probe or the cloud aerosol precipitation spectrometer provide information on cloud particle size distribution and cloud droplet images with fast time resolution (de Reus et al., 2009; Baumgardner

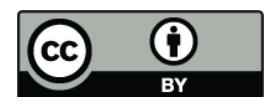

Correspondence to: X.-Y. Yu (xiaoying.yu@pnl.gov) et al., 2001). However, particle morphology and chemical composition measurements are not easily obtained using existing techniques.

Particle mass spectrometers have been used for in-situ particle characterization in recent years (Noble and Prather, 2000; Murphy, 2007; Zelenyuk, 2005). They have fast time resolution, but they cannot observe more than 100 particles per second, which can limit their use when particle concentrations are high. For example, the single particle laser ablation time-of-flight mass spectrometer can detect particles down to $50 \mathrm{~nm}$ at the rate of 20 particles per second (Zelenyuk, 2005). In addition, these aerosol mass spectrometers are rather large and costly instrument platforms. We sought a small, relatively inexpensive device, comparable to our existing time resolved aerosol collector (TRAC) with improved fast particle collection abilities.

Time resolved aerosol collectors have been around for many years. For example, multi-drum sequential impactors (Lundgren, 1967; Raabe et al., 1988) deposit particles via slit nozzles onto rotating drums for post-field laboratory analysis via (usually) PIXE (particle induced $\mathrm{x}$-ray emission) or XRF (x-ray fluorescence). These low-power compact samplers usually are operated with several minutes of time resolution. We developed our own time resolved aerosol collector (TRAC) shown in Fig. 1, optimized for single particle analysis several years ago (Laskin et al., 2003; Hand et al., 2005). The TRAC has been used widely in various field and laboratory studies. This cube-shaped $(17 \mathrm{~cm}$ on a side) device uses a $0.5 \mathrm{~mm}$ nozzle that directs the incoming $0.91 / \mathrm{min}$ airflow against each of the 560 substrates sequentially in the rotating platter. It collects all particles larger than 0.4 microns, and a representative sampling of those down to about $50 \mathrm{~nm}$. These substrates, usually transmission electron microscope grids with thin film coatings of 10 to $100 \mathrm{~nm}$ thick, allow sensitive analysis by automated electron microscopy on a single particle basis. This includes elemental analysis by

Published by Copernicus Publications on behalf of the European Geosciences Union. 


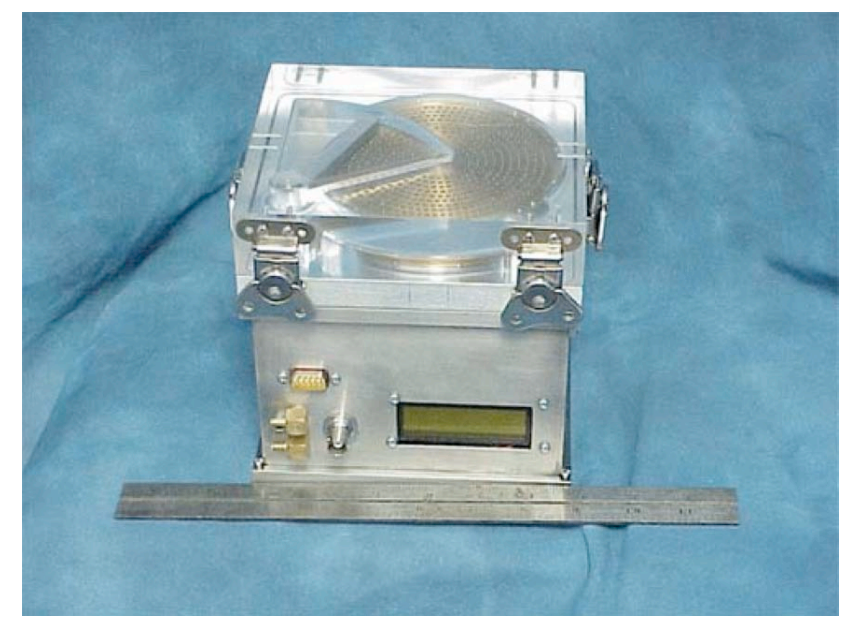

Fig. 1. The existing Time-Resolved Aerosol Collector (TRAC). A 12 inch long ruler is set in front for comparison of size in one dimension.

the energy dispersive $x$-ray spectrum of the particles (Laskin and Cowin, 2001). Its useful time resolution has been about $60 \mathrm{~s}$ per substrate in aircraft studies.

In this work, we demonstrate how to improve the TRAC approach via optical tagging, providing millisecond time resolution. The configuration used to demonstrate feasibility of concept is not field-deployable, and therefore this is a proof of principle paper. We discuss how to make a fielddeployable Fast-TRAC in the end.

\section{Methods}

The particles are collected and optically observed simultaneously. Particles from 0.1 to 10 microns are targeted, as these are commonly found in the atmosphere. Particles in this size range are known to be active as cloud seeding nuclei and of concern for their health effects. Particles smaller than $0.1 \mathrm{mi}-$ cron are also of interest, though detecting these optically can be challenging, as discussed below. The wavelength of light used in this work is larger than many of the particles of interest, and accordingly the internal structure of the smaller particles cannot be resolved. This is not necessary for on-line collection. It is only required that we can detect the particle and know its position adequately to prevent confusion with any neighboring particles. Assume that we have 1-micron resolution, it would suffice to have the density of particles well below 1 particle/1 micron ${ }^{2}$. For example, if 1/25 of this density is assumed, then particles on average are separated by about 5 micron. If the field of view is $1 \mathrm{~mm}$ diameter, then at this density 40000 particles can be detected within the area. This requires at least 1000 by 1000 pixel resolution, to cover as large as $1 \mathrm{~mm}$ at 1-micron resolution.

As research groups routinely use optical microscopy to probe single molecules on surfaces (Xie and Trautman,
1998), detection of $100 \mathrm{~nm}$ particles ought to be tractable based on these successes! Automated optical detection of particles as small as $30 \mathrm{~nm}$ on $20 \mathrm{~cm}$ diameter semiconductor wafer substrates with sub-micron positional accuracy is done routinely (Terrell and Reinhardt, 2008; Li et al., 1991). These applications usually use scanning lasers, and the recent switch to UV lasers has made finding even smaller particles possible. When particles are collected on a nearly featureless substrate, scanning laser methods may be the preferred approach. But when the substrate has significant patterns and contrast, direct imaging methods may be preferable.

Several groups have used scanning or imaging techniques to observe particles while they are being collected. Semleit et al. (1996) experimentally and theoretically analyzed realtime particle detection while particles were deposited onto a far side of a glass plate, as a possible way to examine ambient particles in clean rooms. They concluded that they should be able to detect down to 0.2 micron particles. They were limited by what is considered the "random" scattering of the glass surface, which was at about the $10^{-5}$ to $10^{-6}$ of the incident light. They also concluded that the best ratio of particle signal to substrate scattering involved light scattered at large angles. Mienders et al. (1992) looked at realtime colloid deposition of sub-micron particles with a longrange optical microscope objective. Li et al. (1998) examined micron-sized biological particles sticking to membranes with real-time optical microscopy. Many papers related to these two studies have evolved over time developing "microsieves" and "nanosieves", with goals of using lithography-produced grids for chemical and biological separations. Some have involved real-time observation of particle deposition with automated video analysis (Lin et al., 2009). Another example involves a laser scanner examining particles being deposited over $25 \mathrm{~cm}$ squares, with sensitivity down to at least $0.3 \mathrm{mi}-$ cron particles (Tsuchiya and Takami, 1998).

The detection limit of 0.2 micron particles when adsorbed upon glass (Semleit et al., 1996) resulted from not knowing what the local light scattering of the glass would be. This "random" noise is static. So when (as in our case) we compare sequential frames observing the same area, this static noise can be subtracted, permitting much smaller particles to be detected.

Collecting particles onto a "featureless" substrate (polished glass or silicon) might seem always preferable optically. However, solid substrates are subject to significant haze, and can have significant bulk scattering intensities, making it difficult to observe particles as small as $50 \mathrm{~nm}$ optically (Okamoto, 2006).

With these past experiences and the goal to achieve optimal miniaturization and cost control of an eventual fieldable system, we chose (1) a non-scanning approach (no moving parts), (2) the use of a commercial compact high definition digital video camera for imaging (low cost), and (3) a microsieve particle collection approach (low flow impedance, excellent for post-collection analysis including 


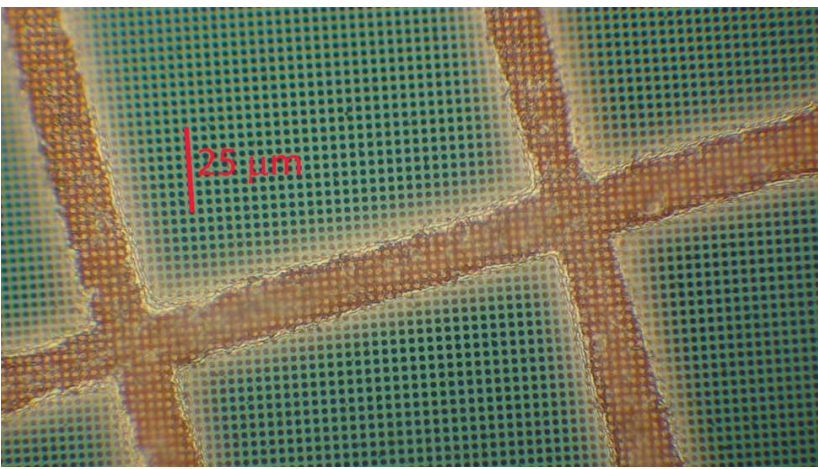

Fig. 2. A Quantifoil substrate imaged with the optical microscope. The legend covers 10 holes or $25 \mu \mathrm{m}$. The fine mesh pitch is $2.5 \mu \mathrm{m}$.

single particle chemical analysis). The evolution of increasingly smaller high resolution imaging systems for consumer products will permit increasingly smaller and cheaper versions of the Fast-TRAC.

The substrates normally used in the TRAC sampler are electron microscope grids that are coated with continuous films tens of nanometers thick. These substrates collect particles by impaction of a jet of air entraining the particles. In this work we use a microsieve-type substrate, which filters out particles by drawing air through it. A Quantifoil substrate is shown in Fig. 2. It is a microlithography-produced $20 \mathrm{~nm}$ thick carbon film, which has orthogonal arrays of 1.2 micron holes with a square 2.5 micron center-to-center distance, on a 200 lines per inch copper grid (Ted Pella, Inc., 658-200$\mathrm{CU})$. The central region of the meshes is very uniform. Being so thin, the substrates have little optical scattering to interfere with optical measurements. They also have low background for electron microscope-based single particle analysis (Laskin and Cowin, 2001). These substrates can be used at a flow of about $31 / \mathrm{min}$ or less to collect $100 \%$ of particles larger than the opening. Particles smaller than the hole size are still collected because their inertia does not permit them to follow the air as it deflects around the openings. For example, around $10 \%$ of the 0.1 micron particles can be collected at $11 / \mathrm{min}$.

The microsieve approach allows elimination of the nozzle, which improves the optical design. For this prototype experiment, a simple holder of the $3 \mathrm{~mm}$ diameter Quantifoil grid was placed under a Zeiss Axioplan microscope (Fig. 3). Air was drawn through the central $2 \mathrm{~mm}$ of the microsieve, and particle containing air was simply introduced by a tube just out of the line of sight, supplying slightly more air than what was drawn through the microsieve. The air flow is expected to be laminar, not turbulent. The Reynolds number for the flow needs to be less than 2300 for the flow to be laminar. Using $11 / \mathrm{min}$ of air flow, over a $2.5 \mathrm{~mm}$ substrate, with a Quantifoil grid of 50\% open area and 2.5 micron openings, then the Reynolds number for the tube flow leading to the grid is about 500 (characteristic length $\approx 2.5 \mathrm{~mm}$ ). When

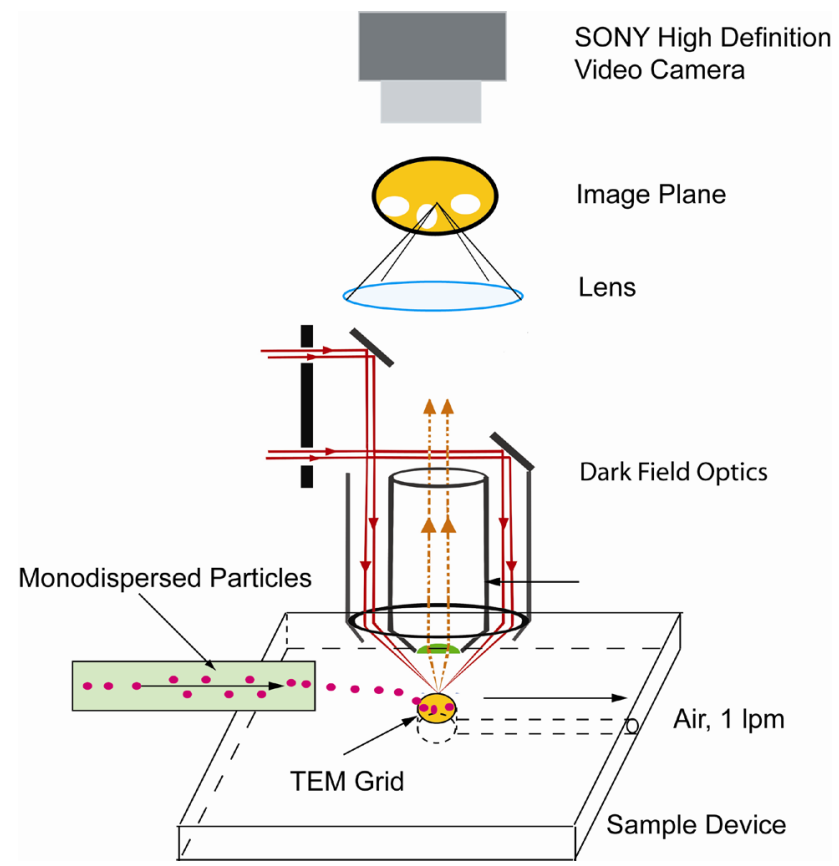

Fig. 3. A schematic showing the proof-of-concept setup of the FastTRAC.

the air is passing around the Quantifoil grid elements, the Reynolds number is about 1 (characteristic length $\approx 2.5 \mathrm{mi}$ cron). So in both locations the flow should be laminar, especially in the most important region, near the grid. The objective was either a 50X or 20X long working distance Neofluar infinity objective. The images were recorded by a compact consumer-oriented Sony HDR-SR7 videocam interfaced to the microscope. The videocam takes $1920 \times 1080$ video at $60 \mathrm{~Hz}$ (interlaced) and 2 megapixel stills. The videocam has a 60 Gigabyte hard drive. The 20X objective with a $12 \mathrm{~mm}$ working distance was sometimes used in imaging, as it provides a larger field of view, yet still adequate resolution. The 50X objective with a $9 \mathrm{~mm}$ working distance was used in illustrating fast framing imaging. The camera also has a $240 \mathrm{~Hz}$ framing mode, for a settable duration (default $=3 \mathrm{~s}$ ).

Particles for the data shown here were polystyrene spherical test particles ranging from $100 \mathrm{~nm}$ to $900 \mathrm{~nm}$ in diameter (Duke Scientific Corporation). Pure or mixed size particles are nebulized from dilute solution into an air stream, dried, and sized with a mobility analyzer (SMPS, TSI 3936) before being directed to the microsieve.

\section{Results and discussion}

\section{Optical tagging at $60 \mathrm{~Hz}$}

Figure 4 shows two sequential frames extracted from several minutes of particle deposition using a $20 \mathrm{X}$ objective. The standard $60 \mathrm{~Hz}$ (60 frames/second) framing rate was used, 

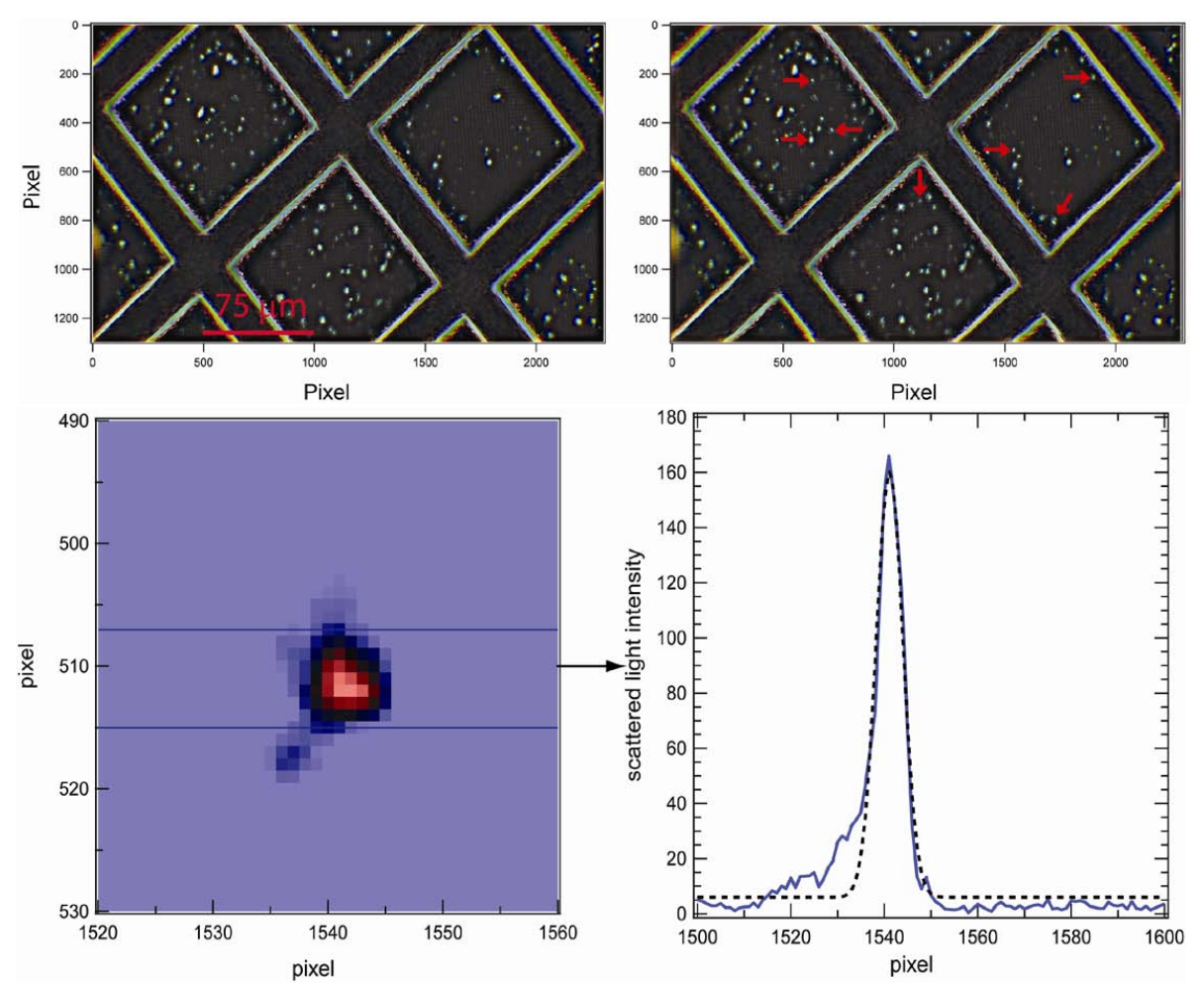

Fig. 4. Two sequential frames obtained at 60 frames/second using the $20 \mathrm{X}$ objective: the first frame (top left) and the consecutive frame (top right). The optical image size is $345.6 \mu \mathrm{m}$ by $194.4 \mu \mathrm{m}$. $200 \mathrm{~nm}$ particles were used. New particles arrived between the two frames are identified with a red arrow in the consecutive frame. Bottom left panel depicts a portion of the difference photo showing a single particle. The profile of this particle is defined by two blue lines. Its scattered light intensity is depicted in the bottom right panel. A Gaussian fit (shown in the dashed line) is used to determine the peak height, width, center, and baseline.

giving about 17 milliseconds between every frame. Several different particles sizes were introduced over this run. The frames shown are from when $200 \mathrm{~nm}$ particles were being deposited. Several single $200 \mathrm{~nm}$ particles land in between those two frames highlighted by red arrows. They are seen in high contrast in the difference image. One near pixel coordinates of 1540 along the $x$-axis and 510 the $y$-axis is shown enlarged in the bottom left panel. The scattered light intensity (as the RGB (red green blue) magnitude) for this particle compared to the background is shown in the bottom right panel of Fig. 4, which is averaged over an 8 pixel high (1.2 micron) line scan. The peak height to background noise is about 80 . A Gaussian fit to the $200 \mathrm{~nm}$ particle image gives a full width half maximum of $600 \mathrm{~nm}$. This is about a wavelength of light (orange). The nominal resolution of the objectives used on the microscope is $340 \mathrm{~nm}$ to $550 \mathrm{~nm}$ (20X and $50 \mathrm{X})$, suggesting that the imaging is near but not quite optimal (thus we should be able to do better yet). When the line scan width is reduced to 4 pixels $(600 \mathrm{~nm})$, to better match a single particle image size, the peak to background ratio increases to about 120 . This shows that we can detect $200 \mathrm{~nm}$ particles with excellent signal to noise. $100 \mathrm{~nm}$ are also routinely detected, as discussed later.
The results indicate that we can identify easily the arrival time of an individual particle to within the framing rate of the camera, and locate it to better than a micron. This is true whether a single particle appears between frames, or whether dozens or even hundreds appear between two frames. If 100 particles appear in 1/60 of a second, this implies a particle rate 6000 particles per second, much faster than can be accommodated by a single particle mass spectrometer. This technique provides us an opportunity to look into events of high particle loadings, such as in an industrial pollution plume, by providing enough resolution of particles in a very short time. The maximum number of particles on the substrate must be kept low enough to prevent confusion in identifying which particle landed at what time, to make it easier to detect the smaller particles, and to make the subsequent single particle analysis straight forward. This means that the average particle spacing should be at least several times a typical particle diameter, as well as several times the optical resolution. This works out to about the same limit, making the maximum permitted number density of particle about $10^{11} / \mathrm{m}^{2}$. For a $2.5 \mathrm{~mm}$ substrate, at $11 / \mathrm{min}$ of airflow, and a particle collection efficiency of $E$, then for a particle loading in the air of $X\left(\right.$ per $\left.\mathrm{m}^{3}\right)$, the time to reach this surface loading is: $10^{11} / \mathrm{m}^{2} /\left(X \times\left(0.001 \mathrm{~m}^{3} / 60 \mathrm{~s}\right) \times E /\left((0.0025 \mathrm{~m})^{2} \times\right.\right.$ 

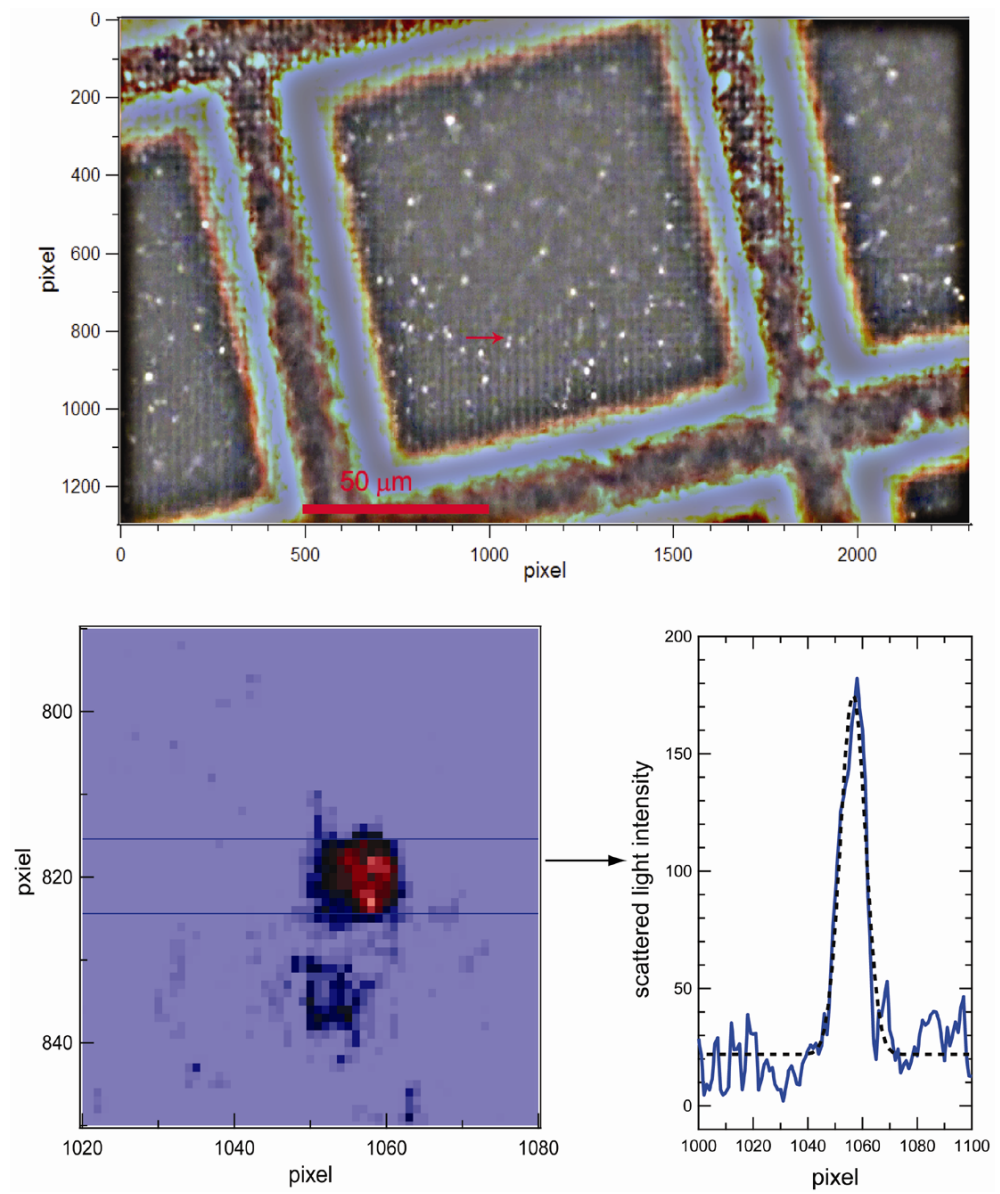

Fig. 5. Illustration of fast framing at 240 frames/second using the $50 \mathrm{X}$ objective. Only the consecutive frame is shown (top). The optical image size is $230.4 \mu \mathrm{m}$ by $129.6 \mu \mathrm{m}$. A single $100 \mathrm{~nm}$ particle arrived between the two frame indicated by a red arrow. The bottom left panel depicts the difference photo of this particle. The profile of this particle is defined by two blue lines. Its scattered light intensity is depicted in the bottom right panel. A Gaussian fit (shown in the dashed line) is used to determine the peak height, width, center, and baseline.

$3.14 / 4))=2.9 \times 10^{10} /(X \times E) \mathrm{s} \mathrm{m}^{-3}$. For $X=10^{11} \mathrm{~m}^{-3}$ (that for a very heavily loaded pollution plume) and $E=0.1$ (typical for our Quantifoil grids), this loading is reached in $2.9 \mathrm{~s}$. This is a fairly short time. However, our TRAC sampler has over 500 substrates in its cartridge. So we could, in real-time, simply move to a new substrate based upon the observed particle density. This would allow about one-half hour of sampling under this extreme condition. If such high loadings were to be typical, it would be simple to dilute the sample air to extend the substrate lifetime. We also note that the particle collection efficiency $E$ using Quantifoil needs to be accurately determined to correctly determine total ambient mass loadings from the collected particle numbers.

\section{Fast framing at $240 \mathrm{~Hz}$}

Figure 5 shows the second of two sequential frames taken at $240 \mathrm{frames} / \mathrm{second}$ using a 50X objective. $100 \mathrm{~nm}$ particles were used in this experiment. A particle that arrived in between the two frames is highlighted by a red arrow. The difference photo depicts the location and scattered light intensity of this single particle at approximately pixel number 1055 along the $x$-axis and pixel number 820 along the $y$ axis. Similarly to the previous analysis using standard framing rate, a line scan is generated for an 8-pixel band $(0.8 \mathrm{mi}-$ cron) in the bottom right panel. The ratio of the peak height to baseline noise is about 9 . These results clearly illustrate that we can easily observe and locate collected particles as small as $100 \mathrm{~nm}$, with a spatial resolution of less than a micron, with a time accuracy of $4 \mathrm{~ms}$.

If we assume that 100 particles arrive in $1 / 240$ of a second using fast framing, this would be 24000 particles per second, which would be handled without difficulty. This optical tagging capability can permit our Fast-TRAC to resolve individual airplane plumes in a single transit from a fast moving airplane. 


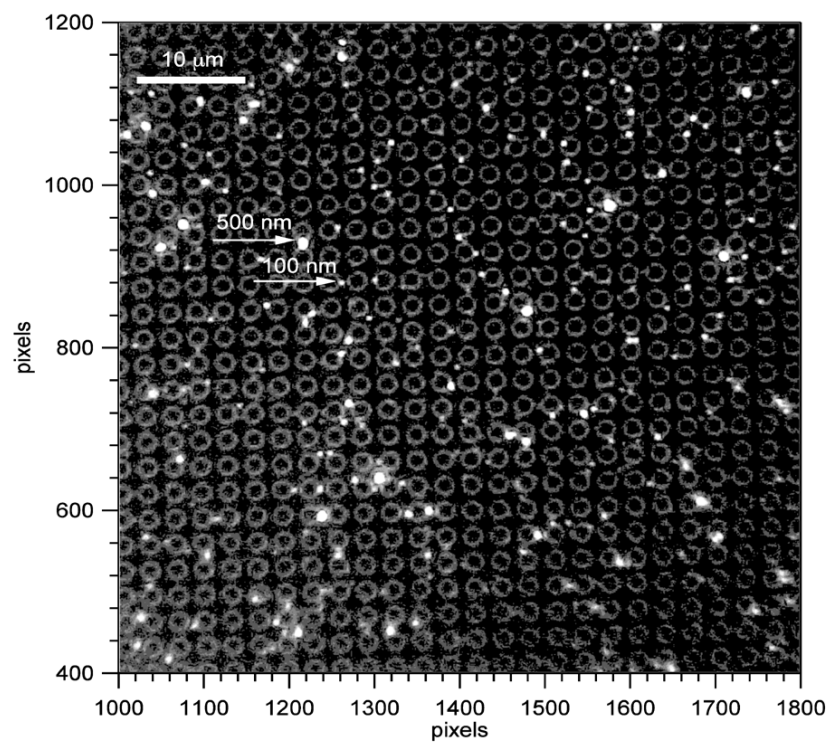

Fig. 6. A still image taken during particle collection showing collected particles of $100 \mathrm{~nm}$ and $500 \mathrm{~nm}$.

In the example shown in Fig. 5, the image is not perfectly focused in all locations. This is because the air going through the filter has bowed the filter inward several microns between the copper supports. Even imperfectly focused we achieved excellent locating ability for $100 \mathrm{~nm}$ particles. Increasing the depth of field will certainly improve the quality of the images and thus the potential field capability of the Fast TRAC. The easiest way to obtain a greater depth of field is to reduce the aperture of the objective. A factor of 3 smaller objective apertures would keep all in focus. However, the reduction of aperture size this much decreases the light intensity by a factor of nine. With the standard illuminator for this microscope, this reduction results in insufficient light. For field deployment, where vibration would also make an increased depth of field valuable, we would opt to illuminate by a diode laser or light emitting diode. Either approach would result in brighter illumination. We could also use blue or near UV diodes, which should increase the brightness for the smallest particles greatly. This is because for particle diameters $(d)$ smaller than the wavelength $(\lambda)$ of light the scattered intensity is proportional to $\pi d^{2} / 4 \times(d / \lambda)^{4}$ (Bohren and Huffman, 1983; Lee et al, 1991).

\subsection{Real-time particle sizing}

Figure 6 shows a sub-region of one frame, where the contrast is adjusted to allow the Quantifoil substrate to be seen. There are at that moment only 100 and $500 \mathrm{~nm}$ particles present. As expected the $100 \mathrm{~nm}$ particles are all of similar brightness, the same is true for the $500 \mathrm{~nm}$ particles. The brighter $500 \mathrm{~nm}$ particles are also discernibly larger than the $100 \mathrm{~nm}$ particles. This is even more obvious in difference images, where the
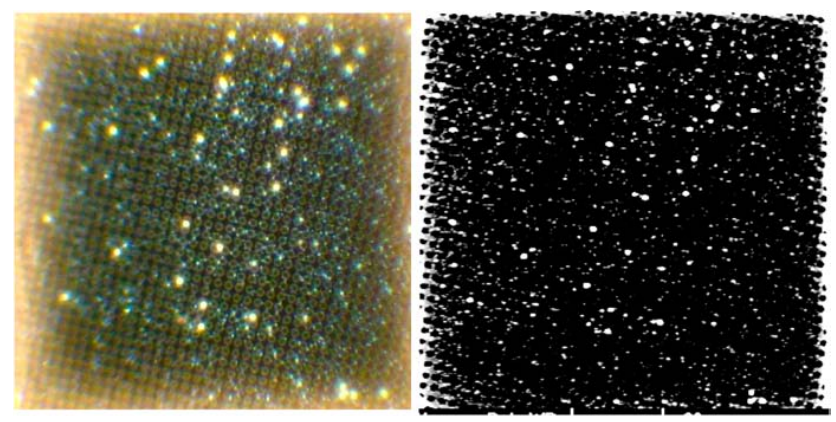

Fig. 7. Comparison of particle identification between the Fast TRAC and the scanning electron microscope. Left is an optical micro-videograph of the substrate with many particles of various sizes taken using the Fast TRAC setup. Right is the same region imaged in a scanning electron microscope.

substrate image is subtracted away. We will use the observed brightness, to turn the images into particle size distributions. This could be done in real-time.

When two particles are very close to each other, they should alter each other's brightness. Similarly, the location of the particle relative to the substrate holes should alter their brightness somewhat (though this is not a strong effect). Figure 5 shows how the presence of a new $100 \mathrm{~nm}$ particle (top and bottom left) makes the pre-existing $100 \mathrm{~nm}$ particle brighter. This pre-existing particle is just over a micron below and to the left of it. In practice we would develop an empirical table to correct for these small brightness changes. Real-time analysis of the difference images to yield in-situ particle distributions seems generally feasible, with a minilaptop programmed for making corrections of adjacency effects.

\subsection{Comparison with SEM images}

Figure 7 shows a region of the grid after exposure to a large number of particles from 100 to $500 \mathrm{~nm}$ in diameter. The left shows the real-time optical image. The right shows the scanning electron microscope (SEM) (FEI XL30) image using a high angle scattering transmission detector. Clearly the images are of the same group of particles. The SEM is used for automated single particle analysis of the elemental composition and particle size distribution. The optical video micrographs permit us to obtain the location of a particle on the substrate and the arrival time of each particle to within $1 / 60 \mathrm{~s}$ $(17 \mathrm{~ms})$ or even $1 / 240 \mathrm{~s}(4 \mathrm{~ms})$ in the field of view. By cross comparison of the two data sets, we obtain the chemical composition of each collected particle with $4 \mathrm{~ms}$ collection time accuracy. For a $200 \mathrm{~m} / \mathrm{s}$ airplane, this corresponds to having a $0.8 \mathrm{~m}$ spatial resolution for obtaining chemical information. This is adequate for exploring cloud microstructures and plumes from a fast moving airplane. 


\subsection{Future directions}

To make a field portable Fast-TRAC, there are a number of challenging technical issues. One of them is making an optical setup that stays in sharp focus despite jarring vibrations by the mobile platform. This can be achieved by making the unit very stiff and small, and likely using automated refocusing. The auto focusing built into the videocam may be adequate. Another issue is optimizing the illumination source of the microscope and increasing the depth of field several fold.

The smallest particles that we might be able to detect are not much smaller than $100 \mathrm{~nm}$. For an $100 \mathrm{~nm}$ particle, the signal to noise ratio is smaller than that of a $200 \mathrm{~nm}$ particle, expected since the scattered light intensity is proportional to the sixth power of the particle diameter for particles smaller than the wavclength (Bohren and Huffman, 1983; Lee et al., 1991), and the exposure time was lower as well. We need to explore the sources of noise where two sequential frames are subtracted. Lower detection limits for particle size may be obtained by using laser diode illumination. Another option is to use the sum of many frames before and after a new particle arrival to artificially increase the exposure time. This will identify smaller particles with reduced time resolution. Full quantitative attention to absolute photo intensities, shot noise, detector readout noise, etc. will be needed to push to the lowest possible particle size. This was not done for this preliminary study. We also did not optimize the objectives used. We get the largest field of view possible on to the camera video sensor using the lowest power microscope objective. This maximizes our sensitivity when the ambient particle density is low. But there are also trade-offs involving light intensity, pixel resolution, and depth of field. In our current set-up, either the 50 or 20 power objective works adequately for most of our purposes. The optimal one may differ when we use different light sources and other transfer optics.

A different approach to improving the limited depth of focus is to operate the microscope as a digital holographic microscope (Antkowiak et al., 2008; Sheng et al., 2006). This involves using a coherent laser diode as the illumination source, and assuring that the image plane has both scattered and unscattered light impinging upon it. This can be done in an imaging mode, where the sample is imaged upon the camera image plane, or slightly out of focus, or even in far field (such as with no lens at all). It will be necessary to compare the two approaches, to see if holography offers advantages.

The microscope optics can be reduced to just containing the objective with standard piezo-drive focusing, a piezo-x-y adjustment, and a single focusing lens to transfer the image to the camera. This can be set into the TRAC configuration shown in Fig. 1, without increasing the overall size (especially since the TRAC unit itself is overdue for re-design, which should shrink the size to about $50 \%$ top to bottom, i.e., $\sim 9 \mathrm{~cm}$ ). In some applications, the total number of particles can fit on a single microsieve grid. In this case, the sample carousel can be eliminated, reducing the size of the sampler even more. In other cases, the carousel version will be used.

Image analysis for particles is well-developed (Ruan, 1992). Classification of particles by size and shape is a classic application of image analysis. Conducting image analysis to follow $1 / 60 \mathrm{~s}$ frame rates is possible, or one can look at every $n$-th frame. This would allow us to display and provide real-time data on the particle count and size, from about $50 \mathrm{~nm}$ to 10 micron. This type of on-line information will allow the sampling microprocessor to change sampling conditions to match the ambient particle loadings, and advance to new substrates automatically, to prevent overloading, during intense particle episodes.

\section{Conclusions}

We demonstrated the feasibility of a fast time-resolved aerosol collector (Fast TRAC). The particle is time-stamped as it arrives at the collection substrate. Its scattered light intensity can be used to determine the size of the particle, with a 4 to $17 \mathrm{~ms}$ time resolution. Post-field microanalysis (e.g. SEM) can be conducted to determine the chemical composition, morphology, and internal structure of each timetagged particle. This new technique can potentially provide us with fast real-time particle detections under various conditions.

Acknowledgements. The work was supported by US Department of Energy under the auspices of the Atmospheric Science Program, under Contract DE-AC05-76RL01830, by the National Oceanic and Atmospheric Administration under the auspices of the Atmospheric Composition and Climate Program under subcontract 47840 , a DOE/BES Chemical Sciences grant (KC-0301020-16248), and an NIEHS Chemical Sensor Program/Gene Environmental Health Initiative (ES016110). The research was performed in the Environmental Molecular Sciences Laboratory (EMSL), a national scientific user facility sponsored by the Department of Energy's Office of Biological and Environmental Research (OBER) and located at Pacific Northwest National Laboratory.

Edited by: J.-P. Putaud

\section{References}

Antkowiak, M., Callens, N., Yourassowsky, C., and Dubois, F.: Extended focused imaging of a microparticle field with digital holographic microscopy, Opt. Lett., 33(14), 1626-1628, 2008.

Bohren, C. F. and Huffman, D. R.: Absorption and scattering of light by small particles, John Wiley \& Sons, Inc., New York, 132 pp., 1983.

Baumgardner, D., Jonsson, H., Dawson, W., et al.: The cloud, aerosol and precipitation spectrometer: a new instrument for cloud investigations, Atmos. Res., 59, 251-264, 2001.

de Reus, M., Borrmann, S., Bansemer, A., Heymsfield, A. J., Weigel, R., Schiller, C., Mitev, V., Frey, W., Kunkel, D., Kürten, A., Curtius, J., Sitnikov, N. M., Ulanovsky, A., and Ravegnani, F.: Evidence for ice particles in the tropical stratosphere 
from in-situ measurements, Atmos. Chem. Phys., 9, 6775-6792, doi:10.5194/acp-9-6775-2009, 2009.

Fan, B., McFarland, A. R., and Anand, N. K.: Aerosol-Particle Losses in Isokinetic Sampling Probe Inlets, Environ. Sci. Technol., 26(2), 390-394, 1992.

Hand, J. L., Malm, W. C., Laskin, A., et al.: Optical, physical, and chemical properties of tar balls observed during the Yosemite Aerosol Characterization Study, J. Geophys. Res.-Atmos., 110, D21210, doi:10.1029/2004JD005728, 2005.

Laskin, A. and Cowin, J. P.: Automated single particle SEM/EDX analysis of submicrometer particles down to $0.1 \mu \mathrm{m}$, Anal. Chem., 73(5), 1023-1029, 2001.

Laskin, A., Iedema, M. J., and Cowin, J. P.: Time-resolved aerosol collector for CCSEM/EDX single-particle analysis, Aerosol Sci. Tech., 37(3), 246-260, 2003.

Lee, H. S., Chae, S., Yan, Y., Pui, D. Y. H., and Wojcik, G. L.: Theoretical and experimental particle size response of wafer surface scanners, Aerosol Sci. Tech., 14(2), 177-192, 1991.

Li, H., Fane, A. G., Coster, H. G. L., and Vigneswaran, S.: Direct observation of particle deposition on the membrane surface during crossflow microfiltration, J. Membrane Sci., 149, 83-97, 1998.

Lin, J., Bourrier, D., Dilhan, D., and Duru, P.: Particle deposition onto a microsieve, Phys. Fluids, 21, 073301, doi:10.1063/1.3160732, 2009.

Lundgren, D. A.: An aerosol sampler for determination of particle concentration as a function of size and time, J. Air Pollut. Control Assoc., 17(4), 225-229, 1967.

Meinders, J. M., Noordmans, J., and Busscher, H. J.: Simultaneous monitoring of the adsorption and desorption of colloidal particles during deposition in a parallel plate flow chamber, J. Colloid Interf. Sci., 152(1), 265-280, 1992.

Murphy, D. M.: The design of single particle laser mass spectrometers, Mass Spectrom. Rev., 26(2), 150-165, 2007.
Noble, C. A. and Prather, K. A.: Real-time single particle mass spectrometry: A historical review of a quarter century of the chemical analysis of aerosols, Mass Spectrom. Rev., 19(4), 248274, 2000.

Okamoto, A., Kuniyasu, H., and Hattori, T.: Detection of 30-40$\mathrm{nm}$ particles on bulk-silicon and SOI wafers using deep UV laser scattering, IEEE T. Semiconduct. M., 19(4), 372-380, 2006.

Raabe, O. G., Braaten, D. A., Axelbaum, R. L., et al.: Calibration studies of the drum impactor, J. Aerosol. Sci., 19(2), 183-195, 1988.

Ruan, J. J.: Measuring particle-size and size distribution from micrographs images, ACS Sym. Ser., 492, 289-304, 1992.

Semleit, D., Fissan, H., and Yu, G.: Detection of deposited particles from the backside of a glass plate, Part. Part. Syst. Char., 13, 34-40, 1996.

Sheng, J., Malkiel, E., and Katz, J.: Digital holographic microscope for measuring three-dimensional particle distributions and motions, Appl. Optics, 45, 3893-3901, 2006.

Stevens, B., Lenschow, D. H., Vali, G., et al.: Dynamics and chemistry of marine stratocumulus - Dycoms-II, B. Am. Meteor. Soc., 84(5), 579-593, 2003.

Terrell, E. and Reinhardt, K.: Detection and measurement of particulate contaminate analysis, in: Handbook of Silicon Wafer Cleaning Technology, 2nd edition, Chapter 8 , edited by: Reinhardt, K. and Kern, W., William Andrew Inc. Publishers, Norwich, NY, 2008.

Tsuchiya, M. and Takami, K.: Instrument for the in situ measurement of depositing particles, Rev. Sci. Instrum., 68, 3161-3167, 1997.

Xie, X. S. and Trautman, J. K.: Optical studies of single molecules at room temperature, Annu. Rev. Phys. Chem., 49, 441-480, 1998.

Zelenyuk, A. and Imre, D.: Single particle laser ablation time-offlight mass spectrometer: an introduction to SPLAT, Aerosol Sci. Tech., 39(6), 554-568, 2005. 